Proceedings of the 16th Czech and Slovak Conference on Magnetism, Košice, Slovakia, June 13-17, 2016

\title{
Magnetization Curves of Geometrically Frustrated Exchange-Biased FM/AFM Bilayers
}

\author{
M. PANKRATOVA* AND M. ŽUKOVIČ \\ Institute of Physics, Faculty of Sciences, P.J. Šafárik University, Park Angelinum 9, 04154 Košice, Slovakia \\ We consider a ferromagnetic/antiferromagnetic bilayer on a triangular lattice in the framework of the classical \\ $X Y$ model. The impact of the geometrical frustration in this system on the magnetization curves and the exchange \\ bias phenomenon is studied. The magnetization curves and the phase diagram for such systems are obtained. We \\ observe horizontal plateaus and a split of the hysteresis loop on the magnetization curves. It is shown that the \\ shift of the hysteresis loop (exchange bias) occurs for the systems with a magnetically hard antiferromagnet.
}

DOI: 10.12693/APhysPolA.131.642

PACS/topics: 75.60.Ej, 75.70.Cn

\section{Introduction}

In this paper we study the exchange bias phenomenon in the ferromagnetic/antiferromagnetic bilayer (FM/AFM) with the geometrical frustration. The exchange bias phenomenon consists in the shift of the magnetic hysteresis loop $M(H)$ along the external magnetic field axis $H$ [1]. Moreover, some experimental studies show the asymmetry of a magnetization curve, the appearance of horizontal plateaus, and the split of hysteresis loops. These effects are widely studied theoretically and experimentally in layered FM/AFM systems but still have no comprehensive explanation. Despite a large number of works, the influence of the geometrical frustration in the bilayer system on the exchange bias has been little studied yet $[2,3]$. The geometrical frustration appears when the minimum of the system energy does not correspond to the minimum of all local interactions. The triangular lattice with the AFM interaction between each pair of spins is a simplest example of the geometrically frustrated system. In this system the frustration appears because of incompatibility between the local interactions and the lattice geometry.

In this paper the FM/AFM bilayers on the triangular lattice are studied in the framework of the classical $X Y$ model with periodic boundary conditions. The outline of the paper is as follows. In Sect. 2 we introduce a layered system made of one FM and one AFM monolayers on a triangular lattice. Section 3 is devoted to the study of the FM/AFM structure with fixed (so-called frozen) AFM magnetic moments. The paper is completed by the concluding remarks.

\section{FM/AFM bilayer on triangular lattice}

In this section we consider a FM/AFM bilayer made of two monolayers on the triangular lattice. The interaction through the FM/AFM interface $J_{1}>0$ is considered to

\footnotetext{
*corresponding author; e-mail: pankratova_mari@mail.ru
}

be ferromagnetic. The exchange interactions in the FM and AFM films are given by the parameters $J>0$ and $J_{0}<0$, respectively. We assume a strong easy-plane anisotropy both in the AFM (layer $A$ ) and FM (layer $B$ ) layers and an additional single-ion anisotropy $\beta_{i}, i=A, B$ in the easy planes of the FM and AFM subsystems. We consider different values of the magnetic anisotropy $\beta_{i}$ for the AFM and FM planes $\beta_{A} \neq \beta_{B}$. It is assumed that the external magnetic field $H$ is directed along the easy axis and can be $H>0$ or $H<0$. The magnetic states of the magnetization vectors in this bilayer are given by the rotational angles $\varphi_{i}$ of spins in the easy plane. The magnetic energy of the system is given by

$$
\begin{aligned}
E & =-J_{0} \sum_{i, j \in A} \cos \left(\varphi_{i}-\varphi_{j}\right)-J \sum_{i, j \in B} \cos \left(\varphi_{i}-\varphi_{j}\right) \\
& -J_{1} \sum_{i \in A, j \in B} \cos \left(\varphi_{i}-\varphi_{j}\right)-H \sum_{i \in A, B} \cos \left(\varphi_{i}\right) \\
& -\frac{\beta_{B}}{2} \sum_{i \in B} \cos ^{2}\left(\varphi_{i}\right)-\frac{\beta_{A}}{2} \sum_{i \in A} \cos ^{2}\left(\varphi_{i}\right),
\end{aligned}
$$

where the first three summations run over all nearest neighbours and the next three over the spins in the respective layers. Assuming spin uniformity in the respective sublattices the possible equilibrium states are given by the equations

$$
\partial E / \partial \varphi_{k, l}=0, \quad(k=1 \ldots 3, l=A, B) .
$$

where indexes $l$ and $k$ correspond to the planes (FM or AFM) and to the sublattices in these planes, respectively. The solutions of these equations are the parallel structures $\left(\varphi_{k, l}=0, \pi\right)$, the non-collinear structures $\left(\varphi_{k, l} \neq 0, \pi\right)$, and the antiparallel structures which correspond to the horizontal plateaus $\left(\varphi_{2, A}=0, \varphi_{2, B}=\right.$ $\left.\pi, \varphi_{k, l}=\pi\right)$, where $k=1,3, l=A, B$, so all magnetic moments are lying along the field except one in the layer $A$ that has an opposite direction. The second type of horizontal plateaus corresponds to a $\varphi_{2, l}=0, \varphi_{k, l}=\pi$, where $k=1,3, l=A, B$.

The transition from the collinear phase to the canted phase corresponds to the bifurcation of the solutions $\varphi_{k, l}=0, \pi$. In the neighbourhood of the bifurcation point, there are canted solutions of Eq. (2) which are in- 
finitesimally close to the collinear states. To find this point, we linearise these equations with respect to the angles $\varphi_{k, l}$ and look for the nonzero solutions of the linearised equations. The analysis of the stability of the $\varphi_{k, l}=\pi$ phase can be done in a similar way.

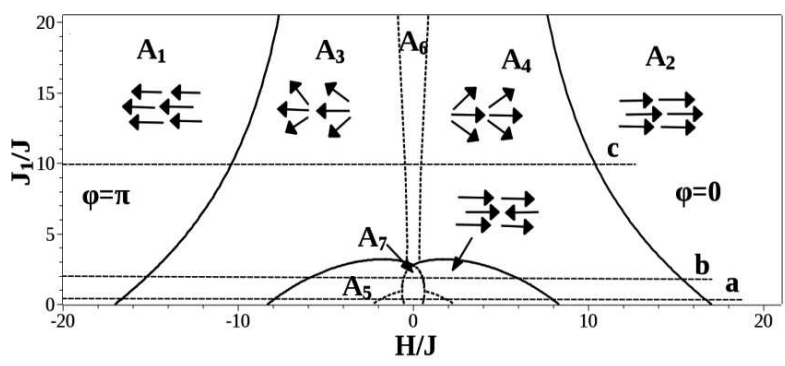

Fig. 1. Phase diagram of the FM/AFM bilayer on the triangular lattice for the values of the parameters $J_{0} / J=-2, \beta_{A} / J=1, \beta_{B} / J=0.5$. The dashed lines correspond to the magnetization curves in Fig. $2 \mathrm{a}-\mathrm{c}$.
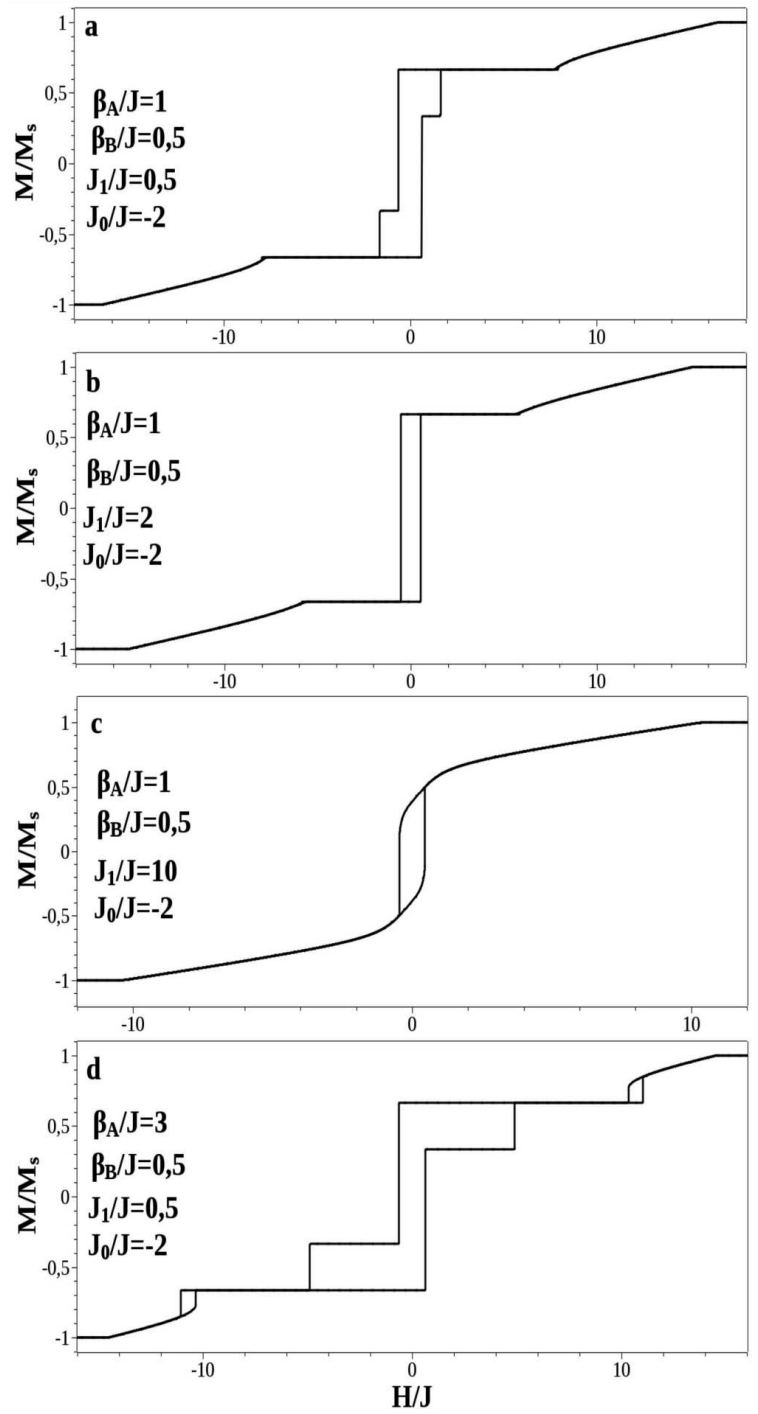

Fig. 2. The hysteresis loops of the $\mathrm{FM} / \mathrm{AFM}$ bilayer for different values of $J_{1}(\mathrm{a})-(\mathrm{c})$ and $\beta_{A}(\mathrm{a}, \mathrm{d})$.
The areas of the existence of the identified structures are given in Fig. 1. The arrows show the direction of magnetization. The parameter regions $A_{1}$ and $A_{2}$ in Fig. 1 correspond to the parallel states in the FM/AFM bilayer, $A_{3}$ and $A_{4}$ to the non-collinear structures, and $A_{5}$ to the existence of the antiparallel states. The small area $A_{7}$ corresponds to the coexistence of horizontal plateaus (see hysteresis loop in Fig. 2a,b,d). The area $A_{6}$ is the domain of the existence of the hysteresis loop in the magnetization curves. The reversible phase boundaries are plotted as solid lines, while the irreversible phase boundaries leading to a magnetic hysteresis are plotted by dotted lines. In the areas $A_{1}, A_{2}$ the external magnetic field is big enough to reverse both FM and AFM and the magnetization vectors in both layers have the same direction. On the contrary, the direction of magnetic moments of the layer $A$ and layer $B$ in the other areas can be different as for example in the horizontal plateau, described above. The lines for the boundaries of the parallel and antiparallel states were obtained analytically from Eq. (2), while the other curves could only be obtained numerically.

The total magnetization of $\mathrm{FM} / \mathrm{AFM}$ bilayer is given by the formula:

$$
M=\sum_{i \in A, B} \cos \varphi_{i}
$$

The hysteresis loops were obtained for different values of $J_{1}$ (Fig. 2a-c) and $\beta_{A}$ (Fig. 2a,d). The split of the hysteresis loop into three loops is observed for some values of the system parameters (Fig. 2d).

\section{The case of frozen AFM}

In this section the AFM layer in the FM/AFM bilayer is considered to be magnetically hard, i.e., for the magnetic fields that are less than the spin-flop transition, its magnetic structure is fixed during the entire magnetization reversal [4]. One can obtain the frozen AFM layer as a surface of a staked AFM with large anisotropy. In this AFM stack the structure of each layer is ferrimagnetic $(\uparrow \downarrow \uparrow)$, with magnetic moments in each layer having opposite direction with their nearest neighbours in the neighbouring layers and thus the total magnetization of the AFM stack is zero. We consider a case of an uncompensated FM/AFM interface. In particular, the collinear structure is considered with two magnetic moments in the triangular plaquette lying opposite to the external magnetic field and one magnetic moment is lying along the field. The uncompensated AFM interface can appear in the following way. Because of the frustration, a noncollinear structure with zero magnetization appears in the AFM layer in zero magnetic field. Due to the interaction with the FM layer the spins in the AFM layer deviate from their original positions and thus form an uncompensated AFM interface [2].

The areas of existence of the parallel $A_{1}, A_{2}$, noncollinear $A_{3}$, and antiparallel $A_{5}$ phases (Fig. 3) and the hysteresis loops (Fig. 4) were obtained in a way similar to that from the previous section. The reversible and 


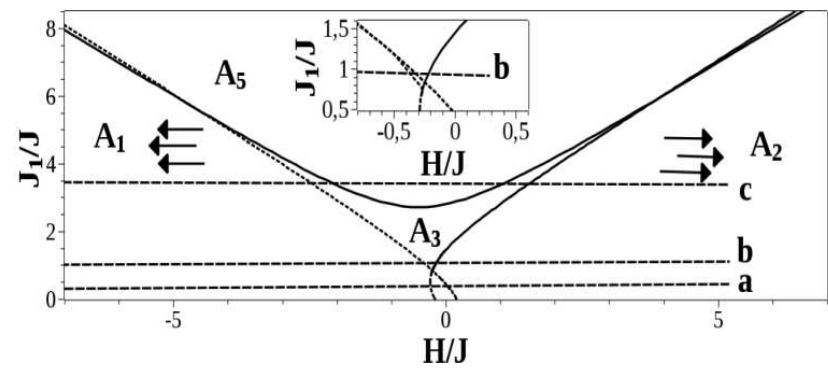

Fig. 3. Phase diagram of the FM/AFM bilayer on the triangular lattice. The case of a fixed AFM for $\beta_{B} / J=$ 0.2 . The dashed lines correspond to the magnetization curves in Fig. 4.
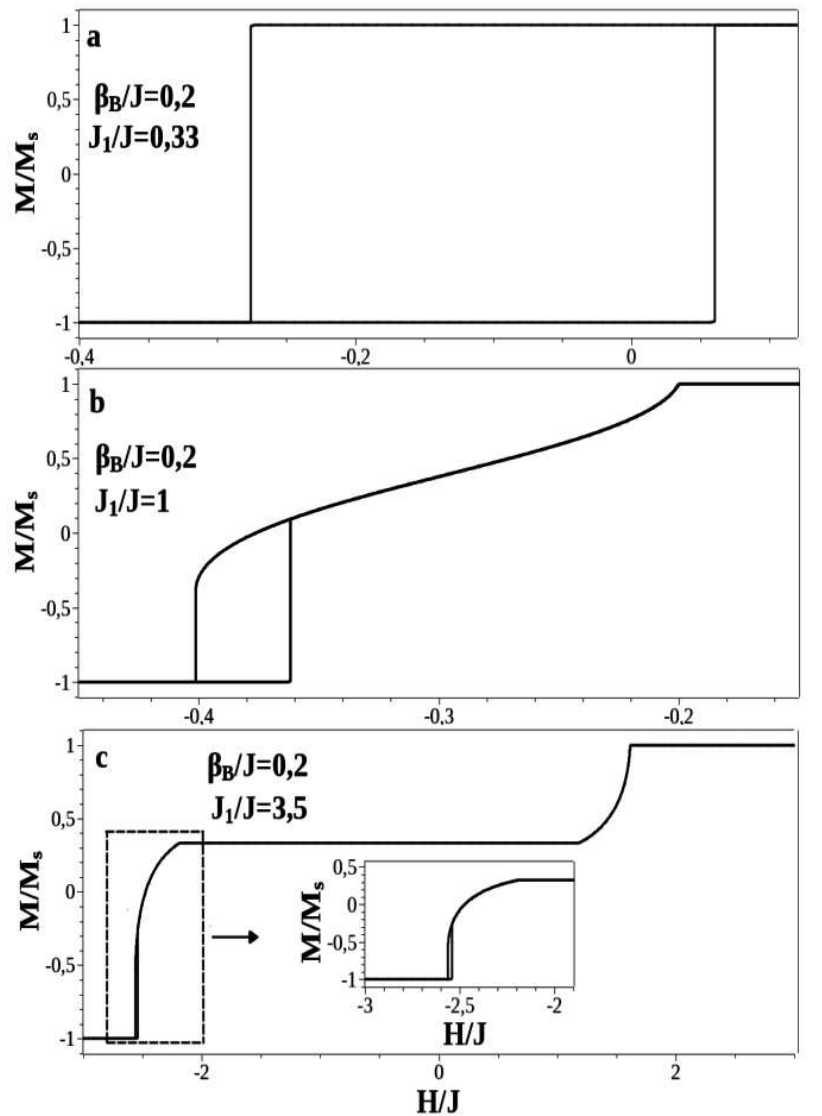

Fig. 4. The hysteresis loops of the FM/AFM bilayer for different values of $J_{1}$. The inset shows a small hysteresis at $H / J \approx-2.5$.

irreversible phase are denoted by the solid and dotted lines, correspondingly. The line which characterise the left boundary of the hysteresis loop (Fig. 4b,c) is almost indistinguishable in Fig. 3 and shown in the inset. It is shown that in this model the shifted magnetization curve can be asymmetric (Fig. 4b,c) and has horizontal plateaus (Fig. 3c). In this section the $M(H)$ curves correspond to the FM layer, while the magnetic moments of AFM are fixed and do not contribute to the magnetization.

\section{Conclusions}

In the framework of the classical $X Y$ model the magnetization curves of the FM/AFM bilayer on a triangular lattice are studied. We have considered the cases of the non-frozen and frozen AFM. The hysteresis loops have been obtained for different values of the exchange interaction and the magnetic anisotropy. The exchange bias is observed in the case of the frozen AFM. Horizontal plateaus and the hysteresis loops are observed for both frozen and non-frozen AFM cases. Phase diagrams have been calculated for selected values of the parameters.

\section{Acknowledgments}

This work was supported by the Scientific Grant Agency of Ministry of Education of Slovak Republic (Grant No. 1/0331/15) and by the National Scholarship Programme of the Slovak Republic.

\section{References}

[1] W.H. Meiklejohn, C.P. Bean, Phys. Rev. 102, 1413 (1956).

[2] C. Mitsumata, A. Sakuma, K. Fukamichi, IEEE, Trans. Magn. 41, 10 (2005).

[3] P. Song, G.K. Li, L. Ma, C.M. Zhen, D.L. Hou, W.H. Wang, E.K. Lui, J.L. Chen, G.H. Wu, J. Appl. Phys. 115, 213907 (2014).

[4] M. Kiwi, J. Mejfa-Lopez, R. Portugal, R. Ramirez, Europ. Lett. 48, 573 (1999). 